\title{
Low Complexity near Lossless Image Compression Technique for Telemedicine
}

\author{
Mohit Gupta \\ National Institute of Technology Raipur, \\ Raipur-492010
}

\author{
Narendra D londhe \\ Assistant Professor \\ National Institute of Technology Raipur, \\ Raipur-492010
}

\begin{abstract}
The minimizations of the storage space and transmission time are the two most important riding factors in image compression for telemedicine. Keeping this in view this paper intend to focus on a comparative investigation of three near lossless image compression technique, NLIC (near lossless image compression), SPIHT with DWT (Discrete Wavelets Transform), RLE (Run Length Encoding) with DCT (Discrete Cosine Transform). These techniques are analyzed and tested on various type of square state of art photographic images and medical images. The performance evaluation parameters like PSNR (Peak Signal to Noise Ratio), CR (Compression Ratio), RMSE (Root Mean Square Error), Computational time (CT) are calculated to evaluate the performance of mentioned near lossless image compression techniques.
\end{abstract}

\section{General Terms}

Image Compression, Telemedicine, Complexity, Near Lossless

\section{Keywords}

Telemedicine, SPIHT, NLIC, RLE, DCT, Huffman Coding.

\section{INTRODUCTION}

Every year, terabytes of medical image data are generated through advance imaging modalities such as magnetic resonance imaging (MRI), ultrasonography (US), computed tomography (CT), digital subtraction angiography (DSA), digital fluorography (DF), positron emission tomography (PET), X-rays and many more recent techniques of medical imaging. These all medical images have wide application in Telemedicine which is the provision of health care services via interactive audio and data communication. It is digitized and computerized process incorporating many technologies like communication, database, and user interface medical science while the foundation of it is communication. As the medical image is very big transmission and storage in medical image often cause difficulty. For example a single 2048x2048 X-ray image may use 4 megabytes and transmitting it over a telephone line operating at 9600 bps may take one hour, which would be very inefficient. So, if we want to get better performance, we'll have either to increase bandwidth of communication channel or to apply compression during transmission. The increase in channel bandwidth may increase the cost of the network and in view of that the work in this manuscript has been focused on image compression during transmission is a good choice.

In the recent decade time, development in image coding research has lead to the emergence of the various lossy and lossless image compression standards. However, storage and transmission of digital images are still demanding for a higher compression rates than ever due increasing bandwidth requirement. Digital images are mainly compressed on the basis of redundancy in two possible ways lossy and lossless. If the exact reconstruction of original image can be achieved, it is called lossless image compression otherwise it is called lossy image compression. In the most recent developments in image coding, researchers have been found concentrating on the methodologies where combined lossy and lossless compression approaches could be used in order to achieve the acceptable compression without sacrificing the signal strength (PSNR). So, on the same line of research the literature has been introduced in the following section.

The lossless image compression is the most desired way of compression but it suffers from a huge drawback of low compression ratio. The latest lossless compression standard JPEG-LS (Yang et al. 2005, Marcos et al., 2008) aims efficient lossless image compression with low complexity. In the view of this several algorithms are proposed like LOCO-I (Low Complexity Lossless Image Compression) (Weinberger et al., 2000), CALIC (Context-based Adaptive Lossless Image Compression) (Wu et al., 2000).

Various forms of image compression techniques mainly taken into consideration for improving performance evaluation parameters which comes under near lossless image compression are stated and describe under the following. The first one known as (Run length Encoding) (Wei Haung et al. 2000) which greatly has been found improving the compression performance and also the security level. This work employs scrambling method which is fast, simple to implement and it provides security. Lossless compression ratios and distortion performance of this method has been found better than other lossless techniques. The second one known as low-complexity embedded compression (EC) algorithm for the JPEG2000 (Chang-Hoon Son et al 2010) which efficiently reduces memory requirements. EC algorithm has reported a fixed compression ratio of $50 \%$ under the nearlossless compression constraint. Most recently, the differential pulse code modulation (DPCM) combined with hierarchical oriented prediction (HOP) (Taquet et al 2010) in order to provide resolution scalability with better compression performances. Moreover, they also have achieved lossless compression results which are about $4 \%$ better than resolution scalable JPEG2000 and close to non scalable CALIC on a large scale database. 
Various application of the above said methods which has wide acceptability in fields like Telemedicine, Tele-consultation (M.A. Ansari et al, 2005), Medical Imaging (Shaou-Gang Miaou et al. 2009).By overlooking at the above image compression scenario, it is found still lagging in terms of complexity of algorithm, compression efficiency and process time which demand further improvements for more storage and faster transmission.

In this paper three near lossless image compression has been investigated one is NLIC (near lossless image compression) which perform initially lossy preparation of image with DCT (Discrete Cosine Transform) followed by lossless Huffman Coding, Second one RLE with DCT which perform initially lossy preparation with DCT followed by lossless run length coding, last one is SPIHT with DWT which perform initially lossy preparation with DWT followed by lossless JPEG encoding based on SPIHT techniques. These techniques are tested on various kinds of square photographic and medical images and compared by evaluating various performance evaluation parameters like compression ratio, peak signal to noise ratio, root mean square error.

\section{PERFORMANCE EVALUATION PARAMETERS}

Mean square error: It is cumulative square difference between original and decompressed image

$$
M S E=\frac{1}{M N} \sum_{u=0}^{M-1} \sum_{v=0}^{N-1}\left(f(u, v)-f^{\prime}(u, v)\right)
$$

Where $f(u, v), f^{\prime}(u, v)$ represent original and decompressed image respectively

Peak Signal to noise ratio: Here signal is the original image and noise is the error in decompression.

$$
P S N R=20 \log _{10}\left(\frac{255}{S Q R T(M S E)}\right)
$$

Compression ratio: Compression Ratio (CR) is defined as number of bit to represent the size of original image to the no of bit to represent the size compressed image. Compression ratio show that how much times the image has been compressed.

$$
C R=\frac{n 1}{n 2}
$$

where $\mathrm{n} 1, \mathrm{n} 2$ represent the number of bit required to represent the original and compressed image. All above parameter are calculated using NLIC technique.

Computational Time: Computational time (CT) is the time required to carry out the compression and decompression operation on the image.

\section{OVERVIEW OF NEAR LOSSLESS IMAGE COMPRESSION TECHNIQUES}

Fig. 1 shows general diagram of low complexity near lossless image compression and decompression techniques. The transformation like DCT, DWT is applied on the gray values of the image and then on transformed gray values entropy encoding like Huffman, RLE, SPIHT techniques is applied for JPEG image compression and for decompression reverse step of above procedure are applied. These technique are discussed as follows

\subsection{Near Lossless Image Compression (NLIC)}

In low complexity NLIC method, firstly the image must be transformed into the frequency domain. The transformed matrix of DCT coefficients of an image is then decomposed in blocks of size $8 \times 8$ and each $8 \times 8$ block of stream line DCT coefficient is encoded by the JPEG standard algorithm based on lossless Huffman coding. In decompression phase of NLIC, the image is reconstructed by applying Huffman decoding followed by IDCT inverse transform applied to the linearly scanned $8 * 8$ block of data.

\subsubsection{Discrete Cosine Transform (DCT)}

The DCT (Suzuki et al. 2010) compression method is a one of the examples of a transform compression. Rather than simply trying to compress the pixel values directly, the image is first transformed into the frequency domain. Compression can now be achieved by more coarsely quantizing the large amount of high-frequency components usually present. This algorithm first break the image into $8 \times 8$ block and scanning from left to right, top to bottom, DCT applied at each block by evaluating the given expression.

$$
\begin{gathered}
D(I, J) \\
=\frac{1}{\sqrt{2 N}} C(I) C(J) \sum_{x=0}^{N-1} \sum_{y=0}^{N-1} g(x, y) \cos \left[\left(\frac{(2 x+1) i \pi}{2 N}\right)\right] \cos \left[\left(\frac{(2 y+1) i \pi}{2 N}\right)\right] \\
C(U)=\left\{\begin{array}{cc}
\frac{1}{\sqrt{2}}, & U=0 \\
1, & U>0
\end{array}\right.
\end{gathered}
$$

DCT is applied at the time of image compression and for decompression of an image reverse process of DCT, i.e. called IDCT (Inverse Discrete Cosine Transform) applied which is calculated by evaluating the given expression

$$
f(I, J)=\sum_{i=0}^{N-1} \sum_{j=0}^{N-1} c(u, v) \cos \left[\left(\frac{(2 i+1) i \pi}{2 N}\right)\right] \cos \left[\left(\frac{(2 j+1) i \pi}{2 N}\right)\right]
$$

where $C(u)$ is same as given above.

\subsubsection{Huffman Coding}

Huffman code procedure is based on the following two observations (Hu et al., 2000).

a. More frequently occurred symbols will have shorter code words than symbol that occur less frequently.

b. The two symbols that occur least frequently will have the same code length. 
The Huffman code (Lakhani, G 2003) is designed by merging the lowest probable symbols and this process is repeated until only two probabilities of two compound symbols are left and thus a code tree is generated and Huffman codes (Bhushan et al., 2009) are obtained from labelling of the code tree. After the code has been created, coding and/or decoding is accomplished in a simple look-up table manner. The code itself is an instantaneous uniquely decodable block code. It is called a block code, because each source symbol is mapped into a fixed sequence of code symbols. It is instantaneous, because each code word in a string of code symbols can be decoded without referencing succeeding symbols. It is uniquely decodable, because any string of code symbols can be decoded in only one way. Thus, any string of Huffman encoded symbols (Howard et al., 1992) can be decoded by examining the individual symbols of the string in a left to right manner.

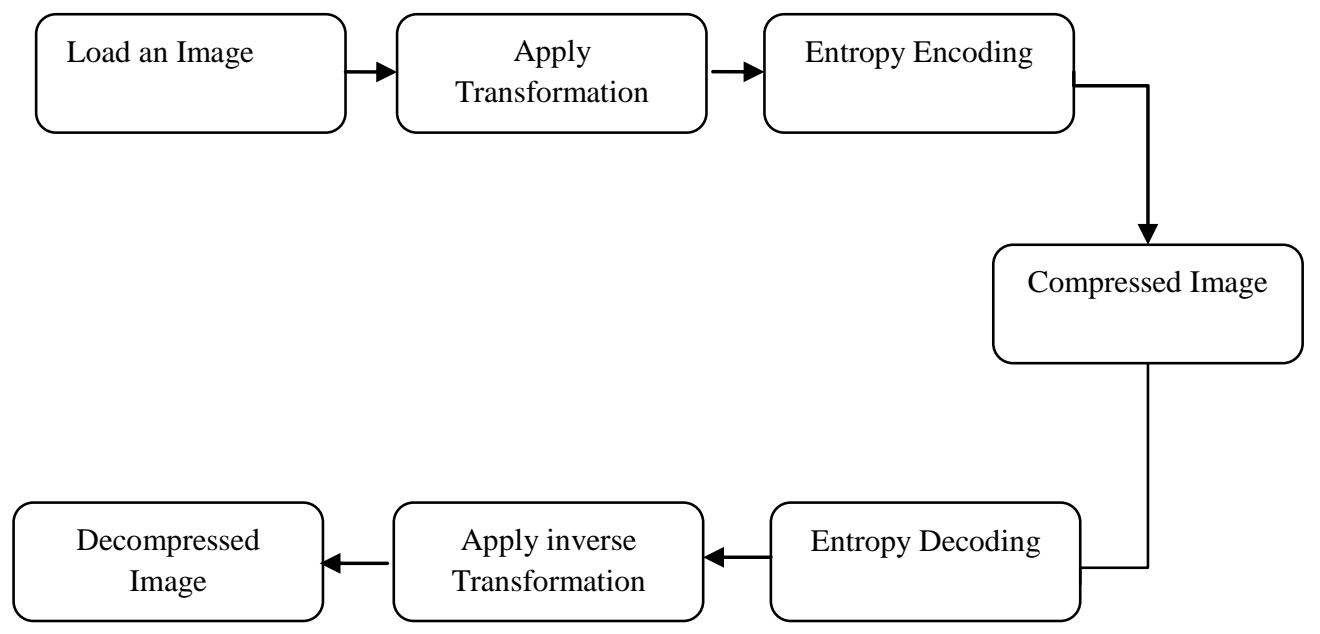

Fig 1: General Flow graph of near lossless image compression and decompression techniques

\subsection{SPIHT-DWT Coding}

SPIHT is well known image compression technique, used for near lossless image compression. SPIHT as probably the most widely used wavelet based algorithm for image compression, providing a standard of comparison for subsequent algorithms.. It first converts the image into its wavelet transform and then transmits information about the wavelet coefficients. The decoder uses the received signal to reconstruct the wavelet and performs an inverse transform to recover the image. We selected SPIHT because SPIHT and its predecessor, the embedded zero tree wavelet coder, were significant breakthroughs in still image compression in that they offered significantly improved quality over vector quantization, JPEG, and wavelets, while not requiring training and producing an embedded bit-stream. SPIHT used three ordered list LIS, LIP, LSP which is defined as LIS List of insignificant sets: contains sets of wavelet coefficients which are defined by tree structures, and which had been found to have magnitude smaller than a threshold (Wen et. al (2005)) (are insignificant). The sets exclude the coefficient corresponding to the tree or all sub tree roots, and have at least four elements. LIP List of insignificant pixels: contains individual coefficients that have magnitude smaller than the threshold. LSP List of significant pixels: pixels found to have magnitude larger that the threshold (are significant)

During the encoding process these LIP, LSP, LIS subsets are examined. A subset becomes labeled as significant if any of its coefficients has a magnitude larger than a given threshold. Like EZW (Thomas et. al (2005)), the significance map encoding (the set partitioning and ordering pass) is followed by a refinement pass, in which the representation of the significant coefficients is refined. Also SPIHT coding can be considered an embedded coding that allows progressive transmission.

\subsection{RLE-DCT Coding}

This technique is quite similar to NLIC. Compressing an image using RLE (et al Al-Wahaib, 2010) is based on the observation of selecting a pixel in the image randomly which tends to make a good chance that its neighbors will have the same gray values. The compressor therefore scans the images row by row, looking for runs of pixels of the same color. If the bitmap starts, e.g., with 17 white pixels, followed by 1 black pixel, followed by 55 white ones, etc., then only the numbers $17,1,55, \ldots$ Need be written on the output stream. The compressor assumes that the images start with white pixels. If this is not true, then the bitmap starts with zero white pixels, and the output stream should start with the run length 0 . The resolution of the images should also be saved at the start of the output stream. The size of the compressed stream depends on the complexity of the image. Resulting that more the detail will present, the worse it will make the compression. The prime goal is to reduce the amount of data needed to be stored or transmitted. RLE algorithms are normally lossless in their operation. However, discarding data during the encoding process, usually by zeroing out one or two least significant bits in each pixel, can increase compression ratios without adversely affecting the appearance of very complex images. This RLE variant works well only with realworld images that contain many subtle variations in pixel values. Making it sure that the RLE encoder always stops at the end of each scan line of bitmap data that is being encoded a lot Also RLE techniques produce varying length of code depending upon the color and content of the image it first like NLIC break an image into $8 \times 8$ block and applying DCT on each $8 \times 8$ block for transformation in frequency domain to coarsely quantize the gray values of the image and then applying run length encoding for producing varying length code and for decompression reverse process of above techniques is applied. 


\section{DIFFERENCE BETWEEN JPEG, NLIC, RLE WITH DCT}

NLIC and RLE with DCT are the variant of JPEG compression with slight modification as Dealing with the $1^{\text {st }}$ step which is to apply transformation on image is almost same in the NLIC, RLE and JPEG. As under JPEG, DCT technique is applied for transforming the image, where as in RLE and NLIC technique same DCT method is applied with a slight difference in the section of scanning the gray value of the images, As per the theory of JPEG, scanning of the image is done in a normal zigzag way where as in this paper linearly scanned the gray values of the image that is starting from the leftmost value to the rightmost value (taking $8 \times 8$ value at a time) which also contributes in reducing the time complexity of the image, as the hoping counts from one pixel to another takes a comparatively less distance in the paper which then proportionate in decreasing time also. Thus the title Low Complexity also gets its justification as the scanning in the very first stage itself is reducing the effort required to compress and decompress.

Taking in concern the $2^{\text {nd }}$ step of RLE, NLIC and the JPEG technique, the JPEG is equipped with the quantization step, which has been removed. As adding quantization step hampers it to be reversible in nature, that is to obtain the real input image value from the compressed gray values becomes tedious. So, the above paragraph throws light on the second difference between the JPEG, NLIC and RLE.T his paper dealing with image transformation only which is used to coarsely quantize the gray values followed by JPEG based encoding and image transformation and JPEG based encoding used to exploit Interpixel redundancy and coding redundancy.

The $3^{\text {rd }}$ step shows the resemblance in both JPEG and our technique as in both the method arithmetic coding is applied to compress and decompress the image.

\section{RESULT}

The result presented in the paper focuses mainly on compression ratio which typically affects the picture quality. Most of the times as researchers go on increasing the compression ratio the quality of the resulting image use to go down. Thus the tradeoff between compression ratio and picture quality is one of the most important aspect to consider when compressing images. The PSNR is most commonly used to measure the quality of reconstruction of near lossless compression and High PSNR of the image means image is reconstructed properly with less MSE.

The tabular comparison is presented in the paper to demonstrate the exact comparison with some numerical proof that NLIC technique supersites the other methods on some performance evaluation parameters like CR, PSNR, CT, MSE. The results in the table are the outcome of the techniques (NLIC and SPIHT, RLE) applied over various standard square photographic and medical images whose results in the form of evaluating performance evaluation parameters like compression ratio (CR), peak signal to noise ratio (PSNR), mean square error (MSE) and Computational time (CT) are shown below in Table 1 and table 2. From table 1, 2 it also makes clear that SPIHT technique gives the fixed reduction (around 10 times) in size of image and also maintain the low complexity which is to be measured by calculating the computational time for compression and decompression of image. Also it suffers from a problem having high average mse and low psnr as compared to other techniques RLE and NLIC. RLE with DCT gives better performance in context of mse and psnr but have a less compression ratio as compared to other two techniques.

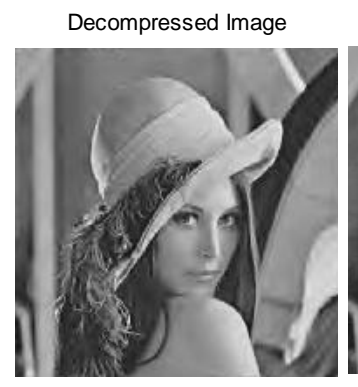

Decompressed image
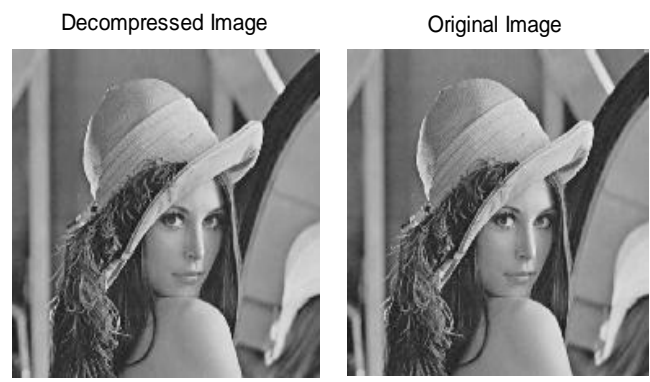

a b

c d

Fig 2: (a) Original Image of size $512 \times 512$ before compression, (b) Decompressed Image using NLIC, (c) Decompressed Image using SPIHT with DWT (d) Decompressed Image using RLE with DCT
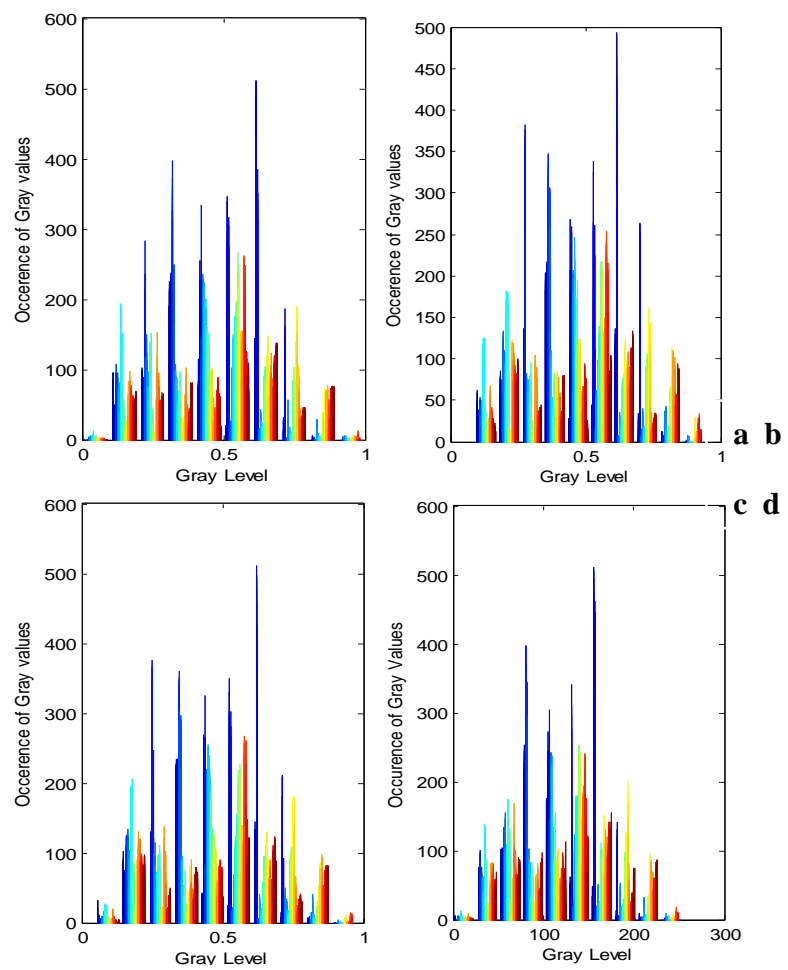

Fig 3: (a) Histogram of Original Image of size 512x512 (b) Histogram of Decompressed Image of NLIC technique (c) 
Histogram of Decompressed Image SPIHT with DWT technique (d) Histogram of Decompressed Image of RLE with DCT

It also suffer from huge disadvantage as size of the image increases than time required to compress and decompress the image will increase, so it unable to maintain the low complexity in context of Computational time. An NLIC technique gives the better performance evaluation parameters parameter of image as compared to other two techniques which are presented in Table1 and Table 2. Fig. 2 (a) shows the original image before compression and Fig. 2(b), 2(c), 2(d) resultant image after decompression using NLIC technique, SPHIT with DCT, RLE with DCT of size $512 \times 512$ respectively.
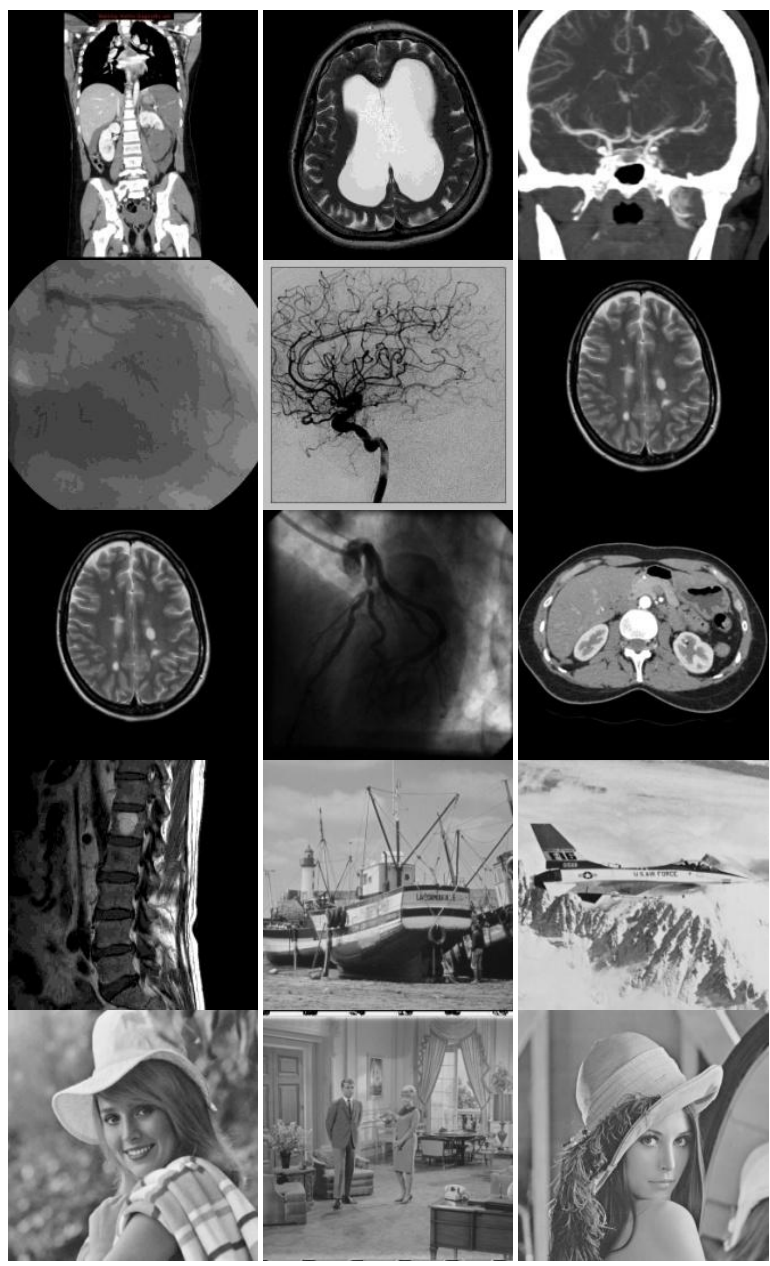

Fig 4: Shows Various Medical and Standard Digital images used in near lossless image compression on which performance evaluation parameters are calculated and presented in Table 1 and Table 2

Fig. 3(a) shows the histogram of original image before compression and Fig. 3(b), 3(c), 3(d) histogram of resultant decompressed image of size $512 \times 512$ using NLIC, SPIHT with DWT and RLE with DCT respectively. From Fig. 3, it is clear that original image and decompressed image are visually undistinguishable but significance loss of information can be observed by plotting the image histograms. But the paper clearly reports in the end that NLIC technique can be acceptable freely where the time is major concern, as depicted in the table that it performs better than the other methodologies.

\section{CONCLUSION}

The results of three near lossless image compression technique are presented and compared that have combined effect of lossless and lossy image compression. NLIC, SPIHT, RLE both techniques basically focuses on reducing the number of bytes to represent an image and can reduce transmission time to send an image over internet or any kind of transmission media with limited channel bandwidth and capacity making the resources more useful. Also the article in its literature part and in the offered results makes it clear that NLIC techniques can achieve high compression ratio with fast decompression time and with high PSNR. It is applied to real volumetric medical image data and can be easily embedded into an online image viewer. Due to the recent advances in CT, MR and nuclear medicine imaging, the size of medical image datasets has increased considerably. This technique attempts to deal with this problem by combining the feature of lossless and lossy image compression to create an optimum solution for transmission of pre-compressed datasets over the Internet. Our experimental results indicate that this solution is much more efficient than the delivery of uncompressed data and that it can greatly improve transmission speed while preserving the quality of the transmitted data. Thus it can be taken as benchmark for the further work to implement other combinations of lossy and lossless image compression techniques. Applying this technique to the remaining various types of images like medical images, radar images etc. can be the recommended work.

\section{REFRENCES}

[1] Al-Wahaib, M.S, KokSheik Wong (2010) 'A Lossless Image Compression Algorithm Using Duplication Run Length Coding', Proc IEEE Conference on Network Application Protocols and Services, pp. 245-250

[2] Avcibas, I, Memon N, Sankur, B, Sayood, K (2002), "A progressive Lossless/Nearlossless imagecompression al gorithm” IEEE Trans: Signal Processing, Vol 9, pp. 312 314 ,

[3] Avcibas, I. Memon, N., Sankur, B, Sayood, K (2005) ,'A Successively refinable lossless image-coding algorithm“ Proc IEEE Trans, Vol 53, PP. 445-452

[4] Bayer, F.M., Cintra, R.J (2010), Image Compression via a Fast DCT Approximation, IEEE Journals of Latin America Transactions, IEEE (Revista IEEE America Latina), Vol 8, pp. $708-713$

[5] Bhooshan, S, Sharma, S. (2009), An efficient and selective image compression schema using Huffman and adaptive interpolation, Inter. Conf. on Image and Vision Computing IVCNZ '09, pp. 197 - 202.

[6] Carvajal, G., Penna, B., Magli, E (2008), Unified Lossy and Near-Lossless Hyper spectral Image Compression Based on JPEG 2000 , journals of IEEE Trans: Geosciences and Remote Sensing, Vol 5, pp. 593-597 
[7] Chai, D, Bouzerdoum, A. (2001), JPEG2000 image compression: an overview , Intelligent Information Systems Conference, pp. $237-241$.

[8] Chang-Hoon Son, Ji-Won Kim, Sung-Gun Song, SeongMo Park, Young-Min Kim (2010), Low complexity embedded compression algorithm for reduction of memory size and bandwidth requirements in the JPEG2000 encoder, journals of IEEE Trans: Consumer Electronics, Vol 56 pp. 2421-2429

[9] Chokchaitam, S., Iwahashi, M .’Lossless, near lossless and lossy adaptive coding based on lossless DCT “ Proc IEEE Conference on Circuit and System, Vol 1, pp. 781-784, 2002

[10] Chokchaitam, S.; Iwahashi, M.(2002) , Lossless, near lossless and lossy adaptive coding based on lossless DCT , Proc IEEE Conference on Circuit and System, Vol 1, pp. $781-784$

[11] Chul Soo Lee, HyunWook Park (2003), Nearlossless/lossless compression of rror-diffused images using a two-pass approach, journals of IEEE Trans: Image Processing, Vol 12, pp. 170-175

[12] Gragory K. Wallace (1991), The JPEG Still Picture Compression Standard, Proc IEEE Trans on Consumer Electronics, Vol 38, pp. 18-34

[13] Howard, P. G., 'Vitter, J.S. (1992) ,Parallel lossless image compression using Huffman and arithmetic coding, Proc. IEEE Data Compression Conf., pp. 299-308. Hu, Y. C., Chang, C.C. (2000) , A new lossless compression scheme based on Huffman coding scheme for image compression, Journal of Signal Processing: Image Communication, Vol. 16, No. 4, pp. 367-372.

[14] Lakhani, G(2003), ,Modified JPEG Huffman coding , journals of IEEE Trans: Image Processing, Vol 12, pp. 159169

[15] M.A. Ansari, R.S. Anand (2008), Recent Trends in image compression and its application in telemedicine and teleconsultation. Proc National System Conferences, pp. $59-64$

[16] Markos E, Papadonikolakis, E., Athanasios P, Kakarountas, E. (2008), Efficient high-performance implementation of JPEG-LS encoder, J. Real-Time Image Proc., Vol. 3, pp.303-310.

[17] Rane, S.D, Sapiro, G (2001) 'Evaluation of JPEG-LS , the new lossless and controlled-lossy still image compression standard, for compression of high-resolution elevation data, journals of IEEE Trans: Geo Science and Remote Sensing, Vol 39, pp. 2298-2306

[18] Sabir, M.F, Sheikh, H.R, Heath, R.W, Jr, Bovik (2006), A joint source-channel distortion model for JPEG compressed images' journals of IEEE Trans: Image Processing, Vol 17, pp. 1349-1364

[19] Shaou-Gang Miaou, Fu-Sheng Ke, Shu-Ching Chen (2009), A Lossless Compression Method for Medical Image Sequences Using JPEG-LS and Interframe Coding, journals of IEEE Trans: Information Technology in Biomedicine Vol 13, pp. 818-821

[20] Suzuki, T., Ikehara, M. (2010) , Integer DCT Based on Direct-Lifting of DCT-IDCT for Lossless-toLossy Image Coding, IEEE Trans. Image Process., Vol.19, pp. $2958-2965$.

[21] Taquet, J, Labit, C. (2010), Near-lossless and scalable compression for medical imaging using a new adaptive hierarchical oriented prediction, IEEE Conferences on Image Processing (ICIP), pp. 481-484.

[22] Thomas W. Fry and Scott A. Hauck (2005) 'SPIHT Image Compression on FPGAs' IEEE Trans. on circuits and systems for video technology, Vol. 15, pp. 1138-1147.

[23] Wei Haung, Shuai Chen and Gengsheng Zheng (2010),Improved Run Length Coding for Gray Level Images Using Gouraud Shading Method, Springer confrence on advance in Wireless Networks and Information System, Vol 72, pp. 19-26

[24] Weinberger, M. J., Sapiro, G., Seroussi, G. (2000) ,The LOCO-I lossless image compression algorithm: principle and standardization into JPEG-LS, IEEE Trans. Image Process., Vol. 9, pp. 1309-1324.

[25] Wen-ChienYen, Yen $\mathrm{Yu}$ chen (2005) 'Natural Image Compression Based on Modified SPIHT ' Proc. IEEE Conference on Annual ACIS International, pp. 100 - 104

[26] Wu, X., Memon, N. (2000) , Context-based lossless interband compression-extending CALIC, IEEE Trans. Image Process., Vol. 9, pp. $994-1001$.

[27] Yang, M., Bourbakis, N. (2005), An overview of lossless digital image compression techniques, Proc. IEEE Circuits and Systems $48^{\text {th }}$ Midwest Symp., Vol. 2, 1099-1102. 


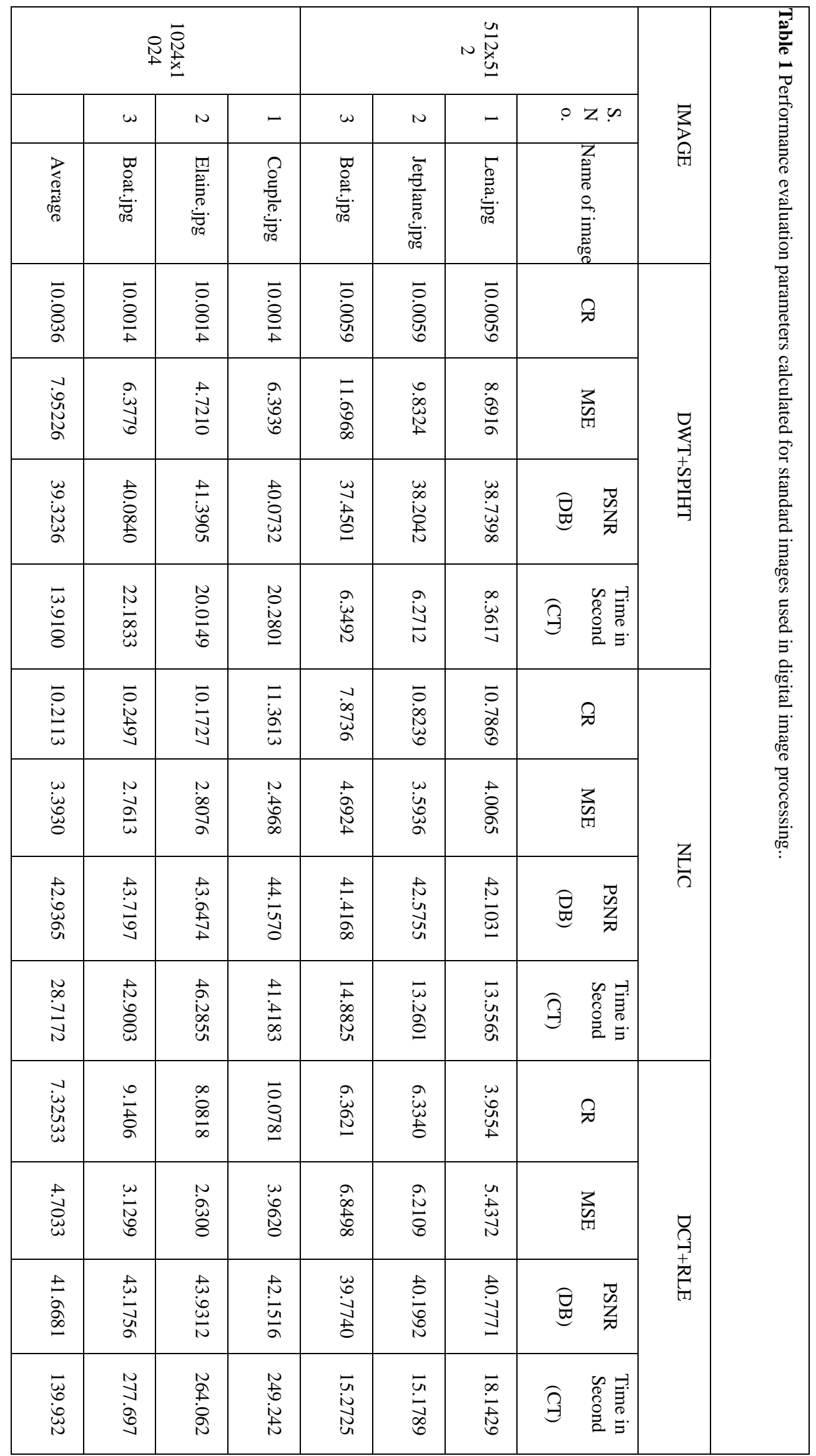




\begin{tabular}{|c|c|c|c|c|c|c|c|c|c|c|c|c|c|c|}
\hline \multicolumn{6}{|c|}{$\underset{\perp}{\mathbb{A}} \vec{x}$} & \multicolumn{7}{|c|}{ N 怣乔 } & \multirow{3}{*}{ 党 } & है \\
\hline & u & + & $\omega$ & $N$ & - & $a$ & u & + & $\omega$ & N & - & $\circ \mathrm{Zn}$ & & $\begin{array}{l}0 \\
0 \\
0\end{array}$ \\
\hline 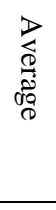 & 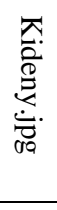 & 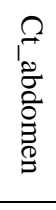 & 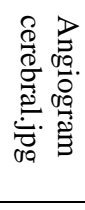 & 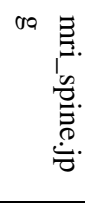 & 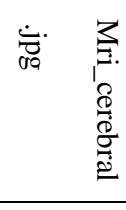 & 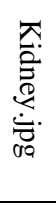 & 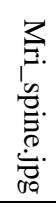 & 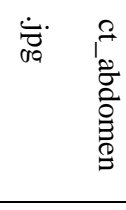 & 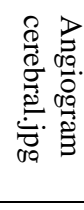 & 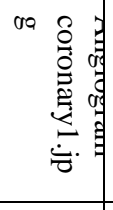 & 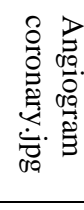 & 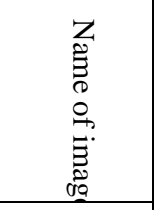 & & 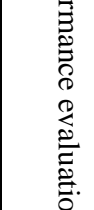 \\
\hline $\begin{array}{l}\text { o. } \\
\dot{0} \\
\text { d్ }\end{array}$ & $\begin{array}{l}\stackrel{0}{0} \\
\dot{8} \\
\end{array}$ & $\begin{array}{l}0 \\
\dot{8} \\
\dot{8} \\
+\end{array}$ & $\begin{array}{l}\stackrel{0}{0} \\
\dot{8} \\
\\
\end{array}$ & $\begin{array}{l}\stackrel{0}{0} \\
\dot{8} \\
\\
\end{array}$ & $\begin{array}{l}\stackrel{0}{8} \\
\dot{8} \\
\end{array}$ & $\begin{array}{l}0 \\
\dot{8} \\
8 \\
0 \\
1\end{array}$ & $\begin{array}{l}\overrightarrow{0} \\
\dot{8} \\
\stackrel{0}{1}\end{array}$ & $\begin{array}{l}0 \\
\dot{0} \\
\dot{8} \\
\text { 1. }\end{array}$ & \begin{tabular}{l}
0 \\
$\dot{0}$ \\
$\dot{8}$ \\
\hdashline \\
1
\end{tabular} & 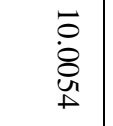 & $\begin{array}{l}0 \\
\dot{8} \\
\dot{8} \\
\infty\end{array}$ & $\approx$ & \multirow{4}{*}{ 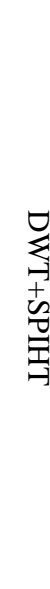 } & 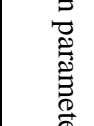 \\
\hline $\begin{array}{l}\text { r } \\
\hat{\sigma} \\
\text { o }\end{array}$ & 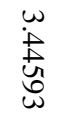 & 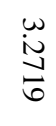 & $\stackrel{N}{\stackrel{n}{\circ}}$ & $\begin{array}{l}n \\
\infty \\
0 \\
0\end{array}$ & $\begin{array}{l}\text { Nu } \\
\text { D̃ } \\
\text { N్ }\end{array}$ & $\begin{array}{l}\text { F } \\
\text { : } \\
\text { 䔰 }\end{array}$ & $\begin{array}{l}\infty \\
\dot{0} \\
\text { ర్ } \\
\infty \\
\infty\end{array}$ & $\begin{array}{l}\ddot{n} \\
\dot{\tilde{\omega}} \\
\ddot{\infty}\end{array}$ & 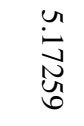 & $\begin{array}{l}\vec{u} \\
\infty \\
+ \\
+ \\
+\end{array}$ & $\begin{array}{l}\stackrel{N}{0} \\
\dot{8} \\
\mathbb{N} \\
\pm\end{array}$ & $\begin{array}{l}3 \\
\text { 至 }\end{array}$ & & 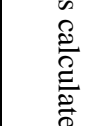 \\
\hline $\begin{array}{l}t \\
\dot{\hat{\alpha}} \\
\text { ô }\end{array}$ & 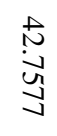 & $\begin{array}{l}\vec{N} \\
\text { ¿ } \\
\stackrel{D}{0} \\
\text { J }\end{array}$ & 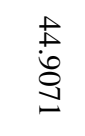 & $\begin{array}{l}+ \\
u \\
\breve{u} \\
\breve{c} \\
u\end{array}$ & $\begin{array}{l}\mathbb{N} \\
\substack{\infty \\
\infty \\
\infty \\
\infty \\
\infty}\end{array}$ & $\begin{array}{l}\text { w } \\
\dot{\omega} \\
\stackrel{\alpha}{a}\end{array}$ & 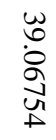 & $\begin{array}{l}\frac{1}{0} \\
\dot{\alpha} \\
\text { oे } \\
\text { à }\end{array}$ & 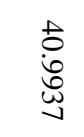 & 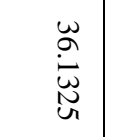 & 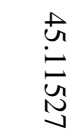 & 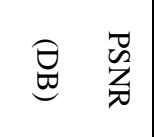 & & \\
\hline $\begin{array}{l}\vec{N} \\
\dot{\omega} \\
\infty \\
\infty \\
+\end{array}$ & $\begin{array}{l}\infty \\
\infty \\
\infty \\
\infty \\
0\end{array}$ & $\begin{array}{l}\dot{\infty} \\
\hat{\sigma} \\
\dot{\delta}\end{array}$ & $\begin{array}{l}\vec{b} \\
\dot{\vec{b}} \\
\dot{\infty} \\
u\end{array}$ & $\begin{array}{l}\overrightarrow{0} \\
\dot{\tilde{U}} \\
\text { \&ै }\end{array}$ & $\underset{\stackrel{N}{\stackrel{N}{y}}}{\stackrel{N}{J}}$ & $\underset{\dot{w}}{\stackrel{w}{\omega}}$ & 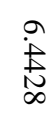 & $\begin{array}{l}\stackrel{a}{\vec{u}} \\
\dot{x} \\
\infty\end{array}$ & $\begin{array}{l}\hat{\mathcal{Q}} \\
\text { 崩 }\end{array}$ & 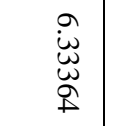 & $\underset{\dot{D}}{\stackrel{D}{\perp}}$ & 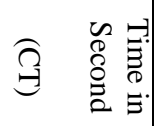 & & \\
\hline $\begin{array}{l}\bar{a} \\
\dot{\omega} \\
u ̈\end{array}$ & $\begin{array}{l}\infty \\
\dot{N} \\
\text { N }\end{array}$ & $\begin{array}{l}\infty \\
+\infty \\
\infty \\
\infty\end{array}$ & $\begin{array}{l}N \\
N \\
0 \\
0 \\
O\end{array}$ & $\begin{array}{l}\infty \\
\infty \\
\dot{\leftrightarrow} \\
\dot{0} \\
+\end{array}$ & $\begin{array}{l}\overrightarrow{0} \\
\dot{0} \\
\dot{\omega}\end{array}$ & $\begin{array}{l}\overrightarrow{0} \\
\dot{\omega} \\
\vec{\omega}\end{array}$ & $\stackrel{\bullet}{\ominus}$ & 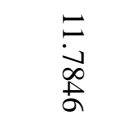 & $\begin{array}{l}\vec{u} \\
\vec{t} \\
t\end{array}$ & $\begin{array}{l}\text { बे } \\
\text { }\end{array}$ & $\begin{array}{l}N \\
\omega \\
0 \\
\sigma \\
\sigma\end{array}$ & 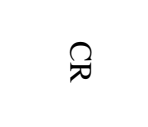 & \multirow{4}{*}{ 2 } & \\
\hline$\underset{N}{w}$ & $\dot{\hat{g}}$ & $\overrightarrow{\tilde{N}}$ & $\underset{\infty}{\vec{J}}$ & 式 & $\begin{array}{l}\dot{\grave{d}} \\
\dot{+}\end{array}$ & 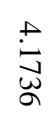 & 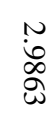 & $\begin{array}{l}\stackrel{n}{a} \\
\dot{b} \\
\stackrel{\infty}{a} \\
\dot{\omega}\end{array}$ & $\begin{array}{l}N \\
\infty \\
\mathbb{d} \\
\delta\end{array}$ & $\begin{array}{l}\stackrel{\vec{w}}{W} \\
\text { a }\end{array}$ & 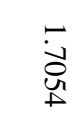 & $\begin{array}{l}3 \\
\text { [1] } \\
\text { [1 }\end{array}$ & & \\
\hline $\begin{array}{l}+ \\
\dot{\omega} \\
\dot{\omega} \\
\dot{\omega}\end{array}$ & $\begin{array}{l}\stackrel{a}{\infty} \\
\stackrel{\infty}{v}\end{array}$ & $\begin{array}{l}\vec{t} \\
0 \\
0 \\
0 \\
+\end{array}$ & 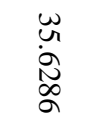 & 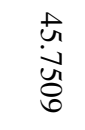 & 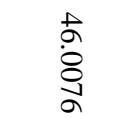 & 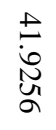 & $\begin{array}{l}\stackrel{+}{\omega} \\
\dot{\omega} \\
\dot{0} \\
+\end{array}$ & 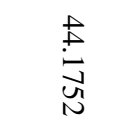 & $\begin{array}{l}\vec{u} \\
\dot{\vec{\Delta}} \\
\dot{\Delta}\end{array}$ & 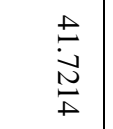 & 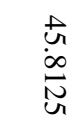 & 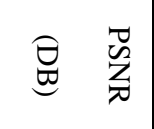 & & \\
\hline 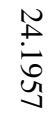 & 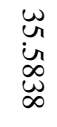 & 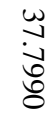 & $\begin{array}{l}\omega \\
+ \\
\infty \\
\ddot{\alpha} \\
\alpha\end{array}$ & 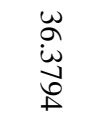 & 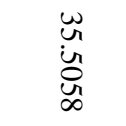 & 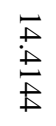 & $\begin{array}{l}\vec{u} \\
i \\
\stackrel{+}{N}\end{array}$ & \begin{tabular}{l}
$\vec{\omega}$ \\
$\dot{0}$ \\
\multirow{+}{+}{} \\
+
\end{tabular} & 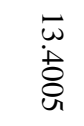 & $\begin{array}{l}\bar{c} \\
\dot{\infty} \\
a \\
\omega \\
\omega\end{array}$ & $\begin{array}{l}\bar{\omega} \\
\dot{\vec{\sigma}}\end{array}$ & 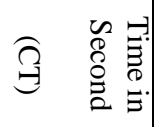 & & \\
\hline oे̀ & $\begin{array}{l}\dot{ن} \\
\dot{0} \\
\dot{0}\end{array}$ & 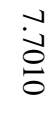 & $\begin{array}{l}\vec{F} \\
\dot{0} \\
\dot{\omega}\end{array}$ & $\begin{array}{l}u \\
\infty \\
\infty \\
\infty \\
a\end{array}$ & $\begin{array}{l}a \\
\dot{0} \\
\stackrel{0}{+} \\
+\end{array}$ & $\stackrel{\omega}{\omega}$ & 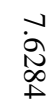 & 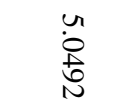 & $\begin{array}{l}\tilde{a} \\
\dot{a}\end{array}$ & $\begin{array}{l}w \\
w \\
w_{1}^{\prime} \\
\dot{w}\end{array}$ & 岕 & 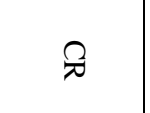 & \multirow{4}{*}{ 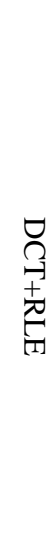 } & \\
\hline 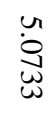 & $\begin{array}{l}n \\
\dot{\omega} \\
0\end{array}$ & $\stackrel{N}{0}_{0}^{0}$ & $\begin{array}{l}N \\
\tilde{W} \\
\infty \\
+ \\
+\end{array}$ & $\underset{\sim}{\vec{u}}$ & 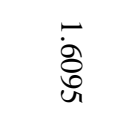 & $\begin{array}{l}\vec{N} \\
\text { U్ } \\
\infty\end{array}$ & $\underset{\infty}{\ddot{\omega}}$ & $\begin{array}{l}\stackrel{9}{\infty} \\
\text { N } \\
\end{array}$ & $\stackrel{\square}{\dot{8}}$ & $\dot{0}$ & $\begin{array}{l}\dot{\infty} \\
\dot{\alpha}\end{array}$ & $\begin{array}{l}3 \\
\text { 齐 }\end{array}$ & & \\
\hline 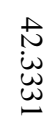 & $\begin{array}{l}+ \\
\dot{\omega} \\
\infty \\
\infty \\
\infty\end{array}$ & 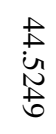 & 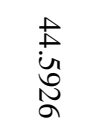 & 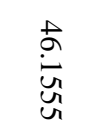 & 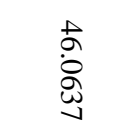 & $\begin{array}{l}\omega_{0} \\
\dot{\mu} \\
\pm \\
\infty\end{array}$ & 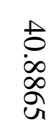 & 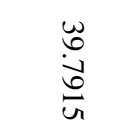 & $\begin{array}{l}\mathscr{w}_{\infty} \\
\stackrel{+}{\infty} \\
\stackrel{\infty}{=}\end{array}$ & 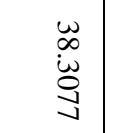 & 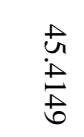 & $\begin{array}{ll}\underbrace{\theta} & \bar{n} \\
\end{array}$ & & \\
\hline $\begin{array}{l}\vec{N} \\
\stackrel{0}{E} \\
\text { E }\end{array}$ & $\begin{array}{l}\tilde{u} \\
\stackrel{4}{+} \\
\dot{\delta}\end{array}$ & $\begin{array}{l}\tilde{N} \\
\tilde{N} \\
\ddot{\omega}\end{array}$ & $\begin{array}{l}N \\
+ \\
\infty \\
1 \\
\stackrel{\omega}{\omega}\end{array}$ & 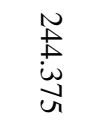 & 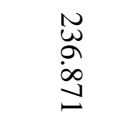 & $\begin{array}{l}\vec{t} \\
\underset{\sim}{\tilde{D}} \\
\infty\end{array}$ & 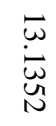 & $\begin{array}{l}w \\
\dot{\infty} \\
\dot{\alpha} \\
\infty\end{array}$ & $\begin{array}{l}\vec{u} \\
\dot{u} \\
\infty \\
\infty\end{array}$ & $\begin{array}{l}\stackrel{+}{+} \\
\stackrel{+}{a} \\
\stackrel{\infty}{\infty}\end{array}$ & $\begin{array}{l}\vec{N} \\
\text { ڤ్ } \\
\text { Oे }\end{array}$ & 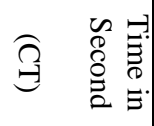 & & \\
\hline
\end{tabular}

